\title{
Numerical and Experimental Study on the Multiobjective Optimization of a Two-Disk Flexible Rotor System
}

\author{
Zheng Longxi, ${ }^{1,2}$ Jia Shengxi, ${ }^{1}$ and Huang Jingjing ${ }^{1}$ \\ ${ }^{1}$ School of Power and Energy, Northwestern Polytechnical University, Xian 710072, China \\ ${ }^{2}$ Collaborative Innovation Center of Advanced Aero-Engine, Beijing 100191, China \\ Correspondence should be addressed to Jia Shengxi; jia_sx@163.com
}

Received 12 October 2016; Accepted 4 December 2016; Published 15 January 2017

Academic Editor: Zuohua Huang

Copyright (c) 2017 Zheng Longxi et al. This is an open access article distributed under the Creative Commons Attribution License, which permits unrestricted use, distribution, and reproduction in any medium, provided the original work is properly cited.

\begin{abstract}
With the ever-increasing requirement for the thrust to weight ratio, the rotational speed of modern aeroengine is increasingly improved; thus most of the aeroengine rotor is flexible. Some dynamic problems, such as excessive vibration, appear due to the increase of the rotation speed of the aeroengine. The aim of this study is to reduce the vibration level of the flexible rotor system through optimum design. A laboratory scale two-disk flexible rotor system representing a typical aeroengine rotor system is designed. A combinational optimization strategy coupling the rotordynamics calculation software ANSYS and the multidisciplinary optimization software ISIGHT is proposed to optimize the rotor system. The positions of the disks are selected as the design variables. Constraints are imposed on critical speeds. The disks' amplitudes and bearings' transmitted forces are chosen as the optimization objectives. Using this strategy, the optimal positions of the two disks are obtained. The numerical optimization results are verified by the experiments based on the test rig. The results show a significant vibration level reduction after optimization.
\end{abstract}

\section{Introduction}

In order to obtain a higher thrust to weight ratio, aeroengines are developed with higher rotation speed and lighter weight, which leads to some critical rotordynamics issues. The most important one is excessive vibration of the rotor system. The excessive vibration may result in fatigue of components and even catastrophic failure. Thus, dynamic design of the rotor system becomes increasingly onerous. Traditionally, the rotor's final structural scheme is obtained through adjusting the structural parameters manually, which is inefficient and has difficulty in achieve the best results [1]. To improve the design quality, shorten the design cycle, and meet the requirements of new generation aeroengine, it is necessary to develop an optimization method through which the final structural scheme of the rotor is determined.

Previous works on the optimum design of rotor system had been done by using transfer matrix method to calculate complex eigenvalues of the system [2-8]. Huang and Luo [2] analyzed the critical speed and unbalanced response of a rotor system using combined method (transfer matrix and pseudomodal method) and changed the stiffness and dampness of the bearing to adjust critical speeds of the rotor. Choi and Yang [3] dealt with the optimum shape design of a rotor shaft. Equations of motion were established and solved to obtain the eigenvalues of the rotor and genetic algorithm was applied to determine the optimum diameters of the rotor shaft. Lee and Choi [4] analyzed a multistepped rotor supported by two angular contact ball bearings by using transfer matrix method and reduced the weight of the rotor through optimization. Huang and Lin [5] obtained the natural frequencies and the critical speeds of a typical rotor bearing system by solving the frequency equation in terms of receptances. The rotor's critical speeds were adjusted through optimizing parameters of supports. A low-pressure steamturbine rotor was analyzed through calculation of eigenvalues and optimized by Yang et al. [6]. Under the constraints of shaft diameter, bearing length, and clearance, the resonance response of the second occurring mode in the excessive vibration was reduced after optimization.

During the design of modern aeroengines, the pursuit of high rotation speed, light weight, and high reliability leads 
to much more complexity of the rotor system. Changing a single design parameter might cause multiple effects on the rotor system [1]. Simulation of rotordynamics and optimum design are becoming increasingly complicated because a large number of parameters need to be considered simultaneously. To solve this, finite element method (FEM) has been used to calculate rotor dynamics to obtain more accurate results on the one hand; multiobjective optimization of rotor system has been launched to fulfill all optimization objectives on the other hand. Minimal weight design optimization of a rotor system was formulated as a nonlinear constrained optimization problem by Pugachev in [9]. An in-house beam-based FEM code was used for the prediction of static and dynamic characteristics of the rotor system and a gradient projection method was used to solve the optimization problem. Using multiobjective optimization, Shiau et al. minimized a geared rotor-bearing system's quality, steady unbalance response, and transmission error response in [10]; Matthew and Patrick reduced a four-supporting rigid rotor's bearing vibration and disk's amplitude in [11]; Choi and Yang minimized the total weight and the transmitted forces at the bearings of a rotorbearing system consisting of a single spool and three bearings in [12].

The previous works have contributed a lot for the optimization of rotor system. However, optimization algorithms and finite element codes used previously were generally inhouse and not widely applicable in engineering rotor optimization. Additionally, the works reported in the literature rarely validated the optimum results through experimental data.

In this paper, a multiobjective optimization strategy based on the combination of commercial finite element software ANSYS and multidisciplinary optimization software ISIGHT is presented. ANSYS is used to calculate the dynamics of rotor system, and ISIGHT is used to solve the optimization problem. The interface between the two types of software is established. A laboratory scale two-disk flexible rotor test rig is established and optimized using the strategy. Experimental verification is carried out on the test rig. The disks' amplitudes and bearings' transmitted forces crossing the first critical speed are reduced significantly after optimization.

\section{Optimization Strategy}

A combined optimization strategy is proposed. The flow chart of the strategy is shown in Figure 1. The procedure of the strategy is as follows.

Step 1 (initial model establishment). This is the first step of the optimization strategy and finished in commercial finite element software ANSYS. It should be noted that the structural parameters of the rotor system which will be selected as optimization variables (parameters that can be changed in optimization, such as positions of bearings, positions of disks, stiffness of bearings, and size of the rotor shaft) should be modeled parametrically. Then dynamics of the rotor system can be obtained.
Step 2 (loading the model in ISIGHT). The two types of software, ANSYS and ISIGHT, are integrated in this step. Simcode assembly in ISIGHT, a command running with input data exchange and output data exchange, is used as the interface between the two types of software.

Step 3 (optimization problem formulation). Based on the dynamic characteristics of the rotor system obtained in Step 1, optimization objectives, design variables, and constraints are determined. The overall objective of the optimization is to reduce the vibration level of the rotor system. Since the most severe deformation occurs at the critical speed and the transmitted forces at two bearings reach the maximum at such a speed, the disks' amplitudes and the bearings' transmitted forces crossing the critical speed are chosen as optimization objectives. As the normal operating speed of the flexible rotor must be apart sufficiently far from its critical speed $[1,13]$, the critical speed should be limited to a certain range. In the present paper, the variation of the critical speed is controlled at the range of $\pm 10 \%$. After this step is completed, the optimization model has been set up. Selecting the optimization algorithm is also completed in this step. It needs to consider both the amount of calculation and the optimization performance when selecting a suitable optimization algorithm for the optimization problem. There are four kinds of multiobjective optimization algorithms in ISIGHT, including Hybrid Multigradient Pareto Explorer (PE), Archive-Based Microgenetic Algorithm (AMGA), Neighborhood Cultivation Genetic Algorithm (NCGA), and Nondominated Sorting Genetic Algorithm II (NSGA-II). In our previous work [14], a rotor system was optimized using those algorithms, respectively, to analyze the performance difference of the algorithms. NSGA-II presented the best optimization performance and acceptable computational cost. So in this paper, NSGA-II is chosen as the optimization algorithm.

Step 4 (DOE (Design of Experiment) analysis). DOE analysis is carried out to determine whether the optimization objectives are sensitive to the design variables and understand the variation tendency of optimization objectives $[15,16]$.

In ISIGHT, the results of DOE are postprocessed using quadratic regression model. The quadratic regression model of optimization problem is established based on sample points:

$$
y=\beta_{0}+\sum \beta_{i} x_{i}+\sum \beta_{j} x_{j}^{2}+\sum_{i \neq j} \beta_{i j} x_{i} x_{j},
$$

where $y$ is the optimization objective, $x$ is the optimization variable, and $\beta$ is the coefficient of the variable. The coefficient represents the main effect of every term (linear term, quadratic term, and interaction term) on the response of $y$. Taking two variables, for example, the regression model is given by

$$
y=\beta_{0}+\beta_{1} x_{1}+\beta_{2} x_{2}+\beta_{3} x_{1}^{2}+\beta_{4} x_{2}^{2}+\beta_{5} x_{1} x_{2} .
$$




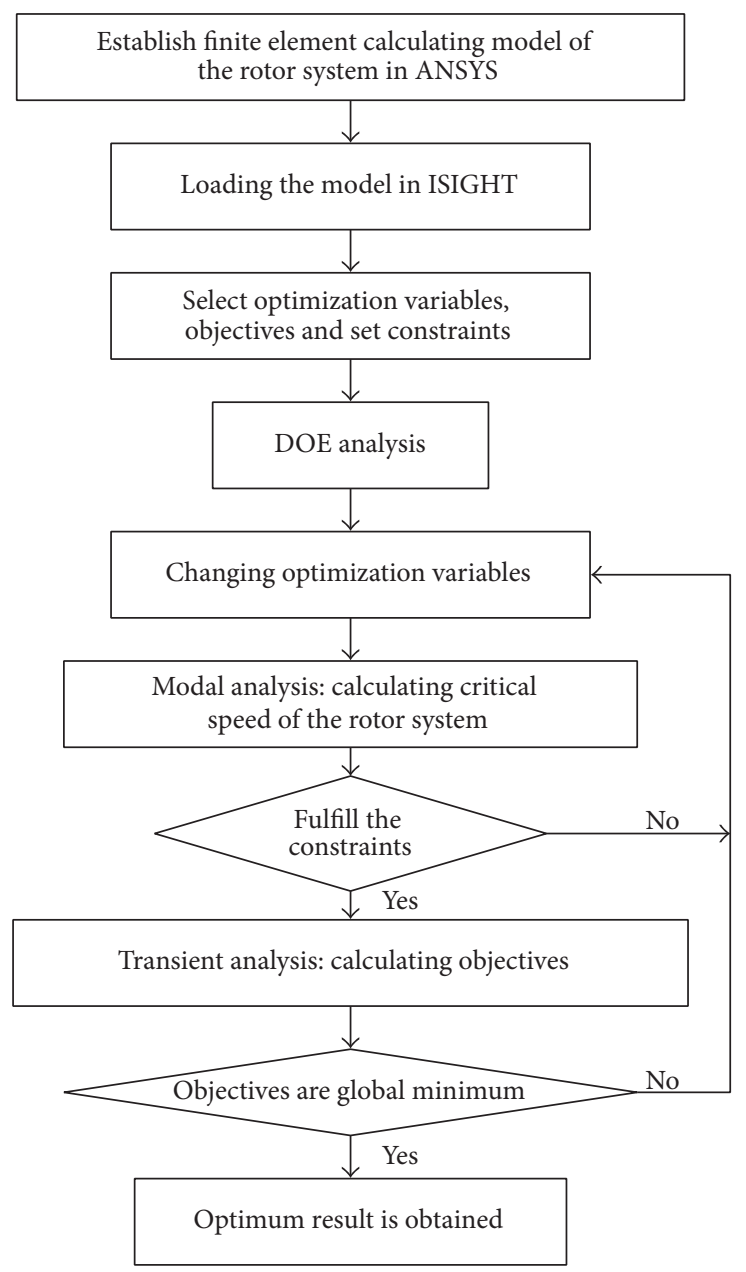

FIGURE 1: Flow chart of the optimization strategy.

The first derivative of $y$ is

$$
\begin{aligned}
d y= & \beta_{1} d x_{1}+\beta_{2} d x_{2}+2 \beta_{3} x_{1} d x_{1}+2 \beta_{4} x_{2} d x_{2} \\
& +\beta_{5} d x_{1} x_{2} .
\end{aligned}
$$

Thus, linear main effect of $x_{1} / x_{2}$ is

$$
\begin{aligned}
& M_{x_{1}}=\beta_{1} d x_{1}, \\
& M_{x_{2}}=\beta_{2} d x_{2},
\end{aligned}
$$

quadratic main effect of $x_{1} / x_{2}$ is

$$
\begin{aligned}
& M_{x_{1}^{2}}=2 \beta_{3} x_{1} d x_{1}, \\
& M_{x_{2}^{2}}=2 \beta_{4} x_{2} d x_{2},
\end{aligned}
$$

and interaction effect of $x_{1} / x_{2}$ is

$$
M_{x_{1} x_{2}}=\beta_{5} d x_{1} x_{2}
$$

where $x, d x$, and $d x_{1} x_{2}$ are calculated as follows:

$$
\begin{aligned}
x= & \frac{[\max (x)+\min (x)]}{2}, \\
d x= & \frac{[\max (x)-\min (x)]}{2}, \\
d x_{1} x_{2}= & {\left[\max \left(x_{1}\right) \min \left(x_{2}\right)+\min \left(x_{1}\right) \max \left(x_{2}\right)\right] } \\
& -\left[\max \left(x_{1}\right) \max \left(x_{2}\right)+\min \left(x_{1}\right) \min \left(x_{2}\right)\right] .
\end{aligned}
$$

Since the range of each variable is different, variables are scaled to $[-1,+1]$ to fairly reflect the contribution of every variable to the response. Then the main effect of every term is fitted by using a least-squares method. The main effect after fitting is presented as Pareto Graph.

Step 5 (iterative calculation). In this procedure, the values of objectives selected in Step 3 are changed according to NSGA-II. Then modal analysis will be performed to obtain the critical speed of the rotor system and to determine whether the critical speed fulfills the constraints. If fulfilled, transient analyses are performed to obtain objectives in current structure and then to determine whether the objectives 


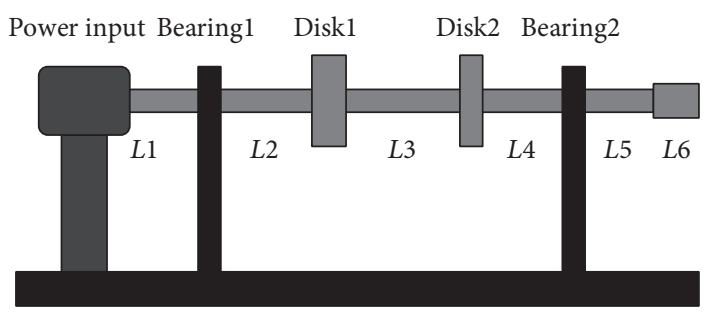

Figure 2: Schematic structure of the two-disk flexible rotor system.

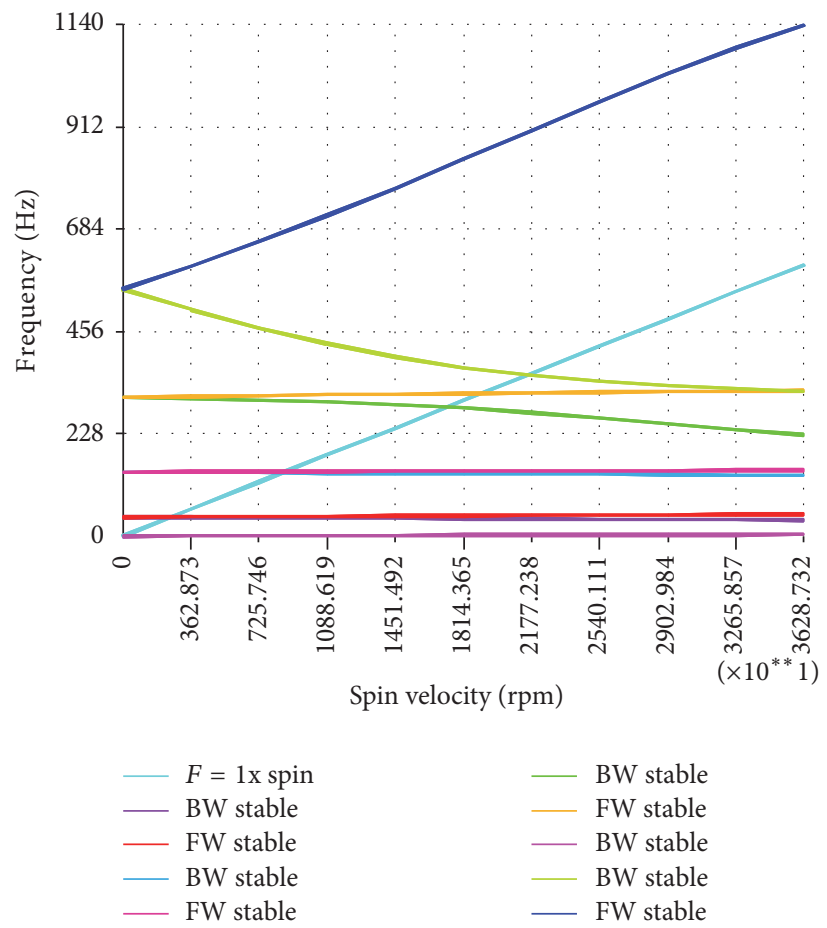

FIGURE 3: Campbell diagram of the rotor system.

are global minimum. If not, the values of variables are rechanged according to NSGA-II. The changed variables are then returned to the ANSYS input file to perform modal analysis and transient analysis again. The procedure is iterated to search possible optimum variables in which objectives become local minimum. After all possible optimum variables (i.e., Pareto front) are obtained, the optimum variables in which objectives become global minimum are chosen from Pareto front.

\section{Optimization of the Two-Disk Flexible Rotor System}

3.1. Two-Disk Flexible Rotor Test Rig. A two-disk flexible rotor test rig is designed and optimized. The schematic structure of the rotor system is shown in Figure 2.

The rotor system is composed of a rotating shaft, two disks (disk1 and disk2), and two supports (bearing1 and bearing2). The maximum speed is $10000 \mathrm{r} / \mathrm{min}$. The diameter of the shaft is $0.009 \mathrm{~m}$. It consists of 6 segments $(L 1=0.0515 \mathrm{~m}$,
$L 2=0.109 \mathrm{~m}, L 3=0.1485 \mathrm{~m}, L 4=0.114 \mathrm{~m}, L 5=0.0355 \mathrm{~m}$, and $L 6=0.034 \mathrm{~m}$ ). The thickness of disk1 is $0.019 \mathrm{~m}$ and its diameter is $0.076 \mathrm{~m}$. The thickness of disk 2 is $0.018 \mathrm{~m}$ and its diameter is $0.075 \mathrm{~m}$. The installation position of disk 1 and disk 2 is $0.1835 \mathrm{~m}$ and $0.332 \mathrm{~m}$ from the left side, respectively (used as a baseline structure). The motor connected at the left side serves as power input of the entire rotor system.

Using rotordynamics calculation software ANSYS, the finite element model of the rotor system is established. Campbell diagram of the rotor system is obtained through modal analysis, which is shown in Figure 3. According to Figure 3, the first-order critical speed of the rotor system (represented by cs1) is $2925.5 \mathrm{r} / \mathrm{min}$. Thus the constraints imposed on the critical speed are

$$
2633 \leq \operatorname{cs} 1 \leq 3218 .
$$

It is worth mentioning that the critical speed can be calculated directly by using ANSYS since the studied rotor system is isotropic. If orthotropic or anisotropic system is considered, ANSYS will be failing to calculate the critical speed; and 


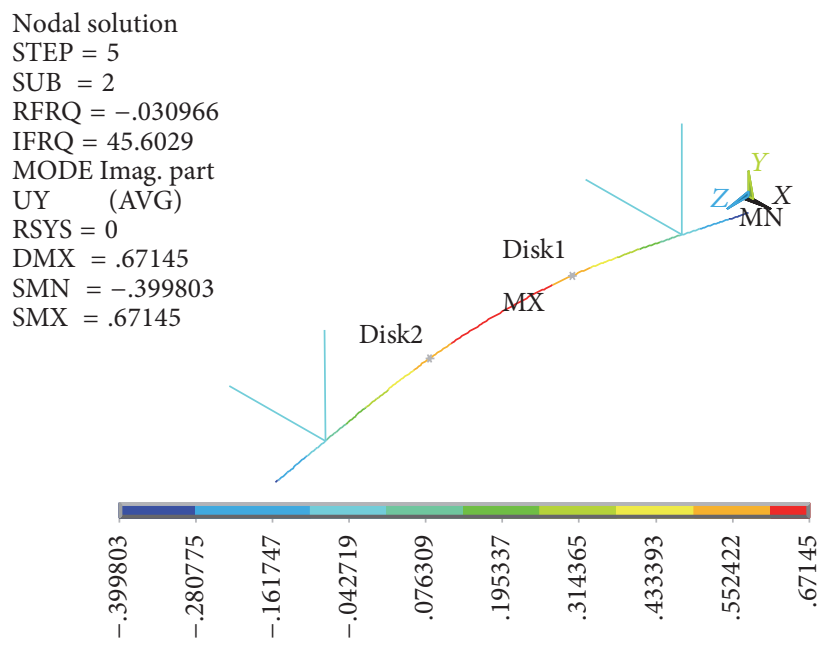

FIGURE 4: The first-order mode shape of the rotor system.

more professional rotordynamics calculation software, such as SAMCEF for ROROR, is recommended.

Figure 4 shows the first-order mode shape of the rotor system. According to Figure 4, the first-order mode shape of the rotor system is flexure type. Reaching the first-order critical speed, the rotor will be flexibly deformed. The most severe deformation occurs at middle part of the rotor where disk 1 and disk 2 are located.

To obtain the dynamic responses of the rotor system, transient analysis (also known as time-history analysis) is carried out. The transient responses of the rotor system strongly depend on the distribution of unbalance mass along the rotor. Since the rotor system is balanced in advance, based upon the experience of balancing, the unbalance mass on disk 1 is assumed $25.5 \mathrm{~g} \cdot \mathrm{mm}$ at 195 degree, and the unbalance mass on disk 2 is assumed $17 \mathrm{~g} \cdot \mathrm{mm}$ at 57 degree. Transient characteristics of the rotor system are shown in Figure 5.

Transient analysis shows the maximum disks' amplitudes and bearings' transmitted forces are reached at the first order critical speed. At such a speed, the amplitude of disk1 and disk 2 is $1.682 e-4 \mathrm{~m}$ and $1.604 e-4 \mathrm{~m}$, respectively, and the transmitted force at bearingl and bearing 2 is $7.8849 \mathrm{~N}$ and $24.93 \mathrm{~N}$, respectively.

The rotor system is then optimized using the proposed strategy to reduce its vibration level. The amplitude of the disk1 and disk2 (represented by al and a2, resp.) and the transmitted force of bearing1 and bearing2 (represented by $\mathrm{f} 1$ and $\mathrm{f} 2$, resp.) crossing the first-order critical speed are chosen as optimization objectives. Positions of the two disks (represented by Disk1_P and Disk2_P, resp.) are chosen as design variables. The rotor system's dynamic performance can be improved by adjusting the distribution of the rotor's mass and stiffness through changing the positions of the disks $[13,17]$.

3.2. Optimization. The initial position of disk 1 and disk 2 is, respectively, $0.1835 \mathrm{~m}$ and $0.332 \mathrm{~m}$ from the left side. Due to the limited moving range of disks in actual aeroengine rotor system, the position of disk 1 and disk 2 is, respectively, left and right moving $0.01 \mathrm{~m}$ from initial position (i.e., Disk1_P changes in $0.1735 \mathrm{~m} \sim 0.1935 \mathrm{~m}$; Disk2_P changes in $0.322 \mathrm{~m} \sim$ $0.342 \mathrm{~m}$ ). Pareto Graphs obtained from DOE analysis are shown in Figures 6 and 7.

Figure 6 shows the effect of Disk1_P on optimization objectives. Figure 7 shows the effect of Disk2_P on optimization objectives. The values of every term in Pareto diagram are the main effect obtained from (4)-(7) after fitting. In Pareto Graph, the blue means positive effect and the red means negative effect [15]. If the proportion of linear term is greater than that of quadratic term, the relationship between variables and objectives is dominated by linear relationship.

Figure 6 shows that there is an approximately linear relationship between the position of diskl and all of the objectives: decreasing the value of Disk1_P the value of al, $\mathrm{a} 2, \mathrm{fl}$, and $\mathrm{f} 2$ decreases, which is desirable in a minimization problem. Therefore, Disk1_P should be reduced in order to reduce a1, a2, f1, and f2. According to Figure 7, a1, f1, and f2 are increased as Disk2_P increases from $0.322 \mathrm{~m}$ to $0.342 \mathrm{~m}$; however, a 2 are decreased. That is to say, reducing Disk2_P can lead to a decrease in $\mathrm{al}, \mathrm{f} 1$, and $\mathrm{f} 2$ and an increase in $\mathrm{a} 2$.

In the present multiobjective optimization, the relative importance of each objective is of the same level, which means all the objectives should be minimized. In order to decrease the values of all the objectives, the values of Disk1_P and Disk2_P have a decrease tendency in the optimization process according to Figures 6 and 7. However, if the values of Disk1_P and Disk2_P are decreased to their minimum, the values of al, $\mathrm{f} 1$, and $\mathrm{f} 2$ are decreased unsurprisingly, but the value of a 2 may be increased because of the relationship of a2 and Disk2_P. To determine the optimum values of Disk1_P and Disk2_P, multiobjective optimization is carried out using NSGA_II. Optimization result is shown in Table 1.

As can be seen from Table 1, the values of al, a2, f1, and $\mathrm{f} 2$ are decreased by $11 \%, 2 \%, 5 \%$, and $8 \%$, respectively, after optimization. What is more, the values of Disk1_P and Disk2_P are decreased but not reaching their minimum, which agrees well with the DOE analysis results. 


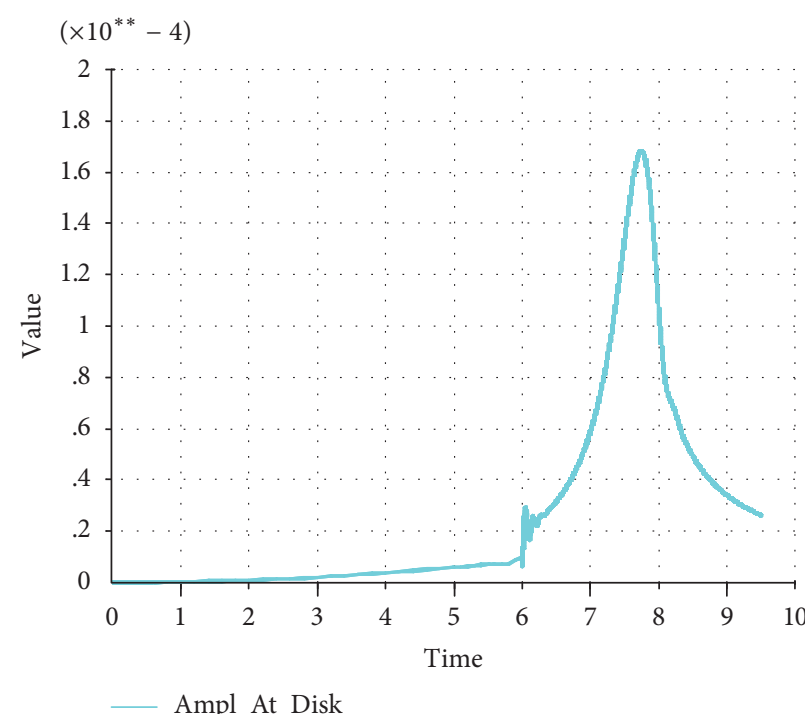

(a) Amplitude of disk1 (al) versus speed

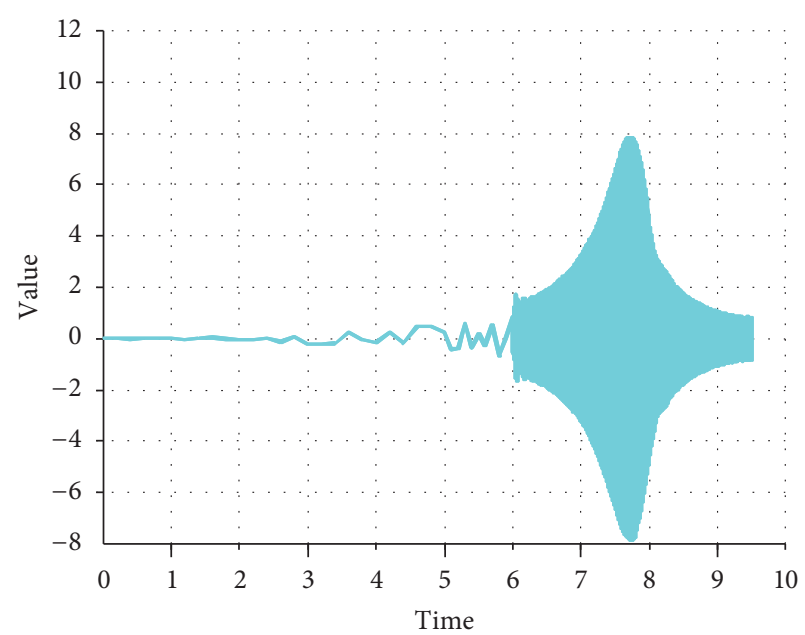

FX_1

(c) Transmitted force of bearingl (f1) versus speed

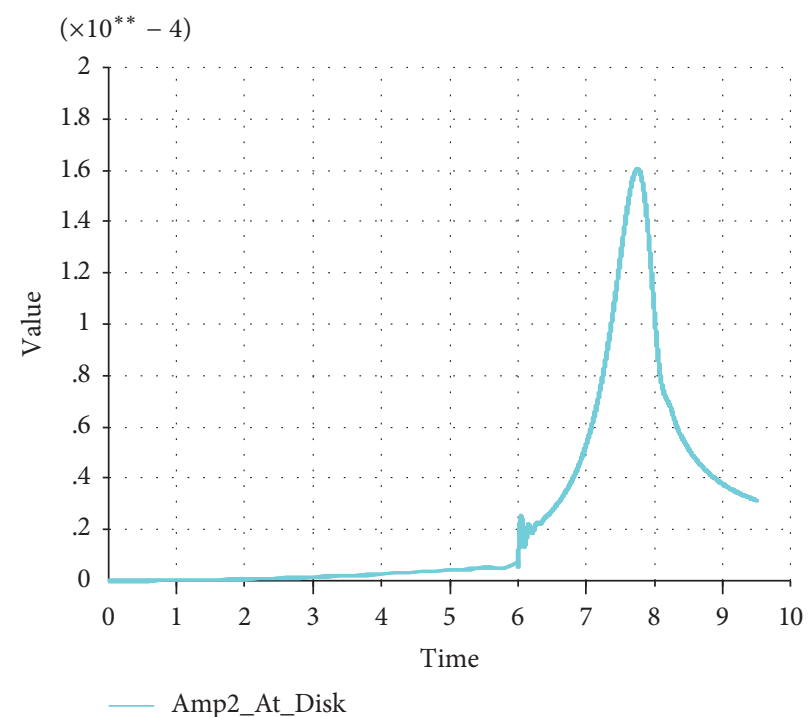

(b) Amplitude of disk2 (a2) versus speed

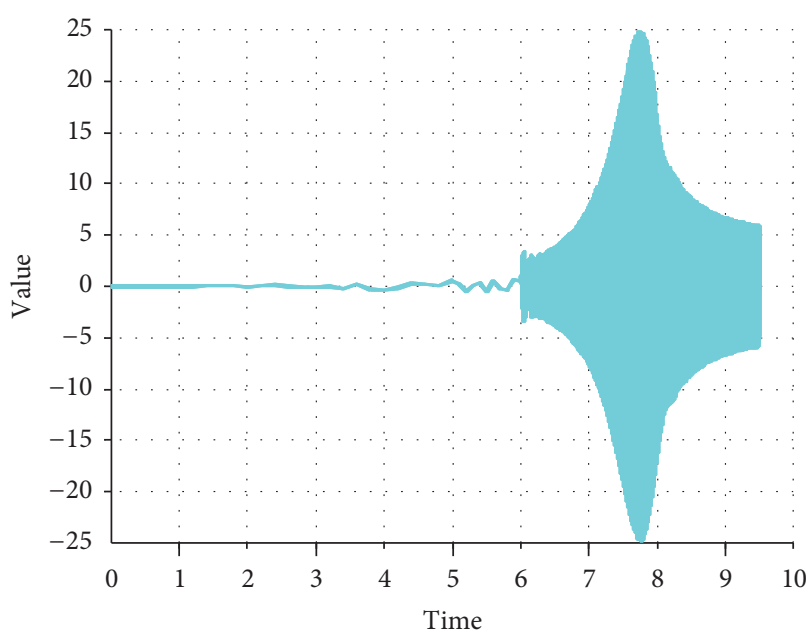

- FX_2

(d) Transmitted force of bearing2 (f2) versus speed

FIgURE 5: Transient responses of the rotor system.

TABLE 1: Numerical optimization result of Disk1_P and Disk2_P.

\begin{tabular}{lccccccc}
\hline & Disk1_P/m & Disk2_P/m & al/e $-4 \mathrm{~m}$ & a2/e $-4 \mathrm{~m}$ & $\mathrm{f} 1 / \mathrm{N}$ & $\mathrm{f} 2 / \mathrm{N}$ & $\mathrm{cs} 1 /(\mathrm{r} / \mathrm{min})$ \\
\hline Baseline & 0.1835 & 0.332 & 1.682 & 1.6044 & 7.8849 & 24.93 & 2926 \\
After optimization & 0.178 & 0.324 & 1.5 & 1.58 & 7.47 & 23.07 & 2851 \\
\hline
\end{tabular}

\section{Experimental Results and Discussion}

To verify the validity of the numerical optimization results, experiments are carried on the test rig shown in Figure 8.

A photoelectric sensor is used to capture the rotor shaft's rotation speed, two TR81 eddy current displacement sensors are used to measure the two disks' amplitudes, and two integrated circuit piezoelectric acceleration sensors are used to measure the two bearings' accelerations.

Move Disk1_P from initial $0.1835 \mathrm{~m}$ to optimized $0.178 \mathrm{~m}$ and move Disk2_P from initial $0.332 \mathrm{~m}$ to optimized $0.324 \mathrm{~m}$.
Amplitudes of the disks and transmitted forces at the bearings before and after optimization are compared in Figure 9.

The longitudinal axis of Figures 9(c) and 9(d) is voltages of the bearings' vibration acceleration which are directly measured by the acceleration sensors. Because there is a linear relationship between the voltages of the vibration acceleration and the magnitudes of transmitted forces at the bearings, such voltages are used to characterize the vibration of bearings.

In Figure 9, dashed lines represent the transient response before optimization and solid lines represent the response 


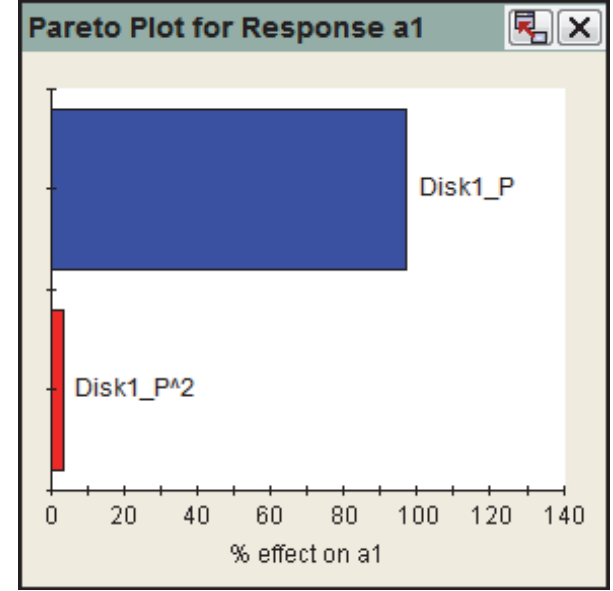

(a) Effect on al

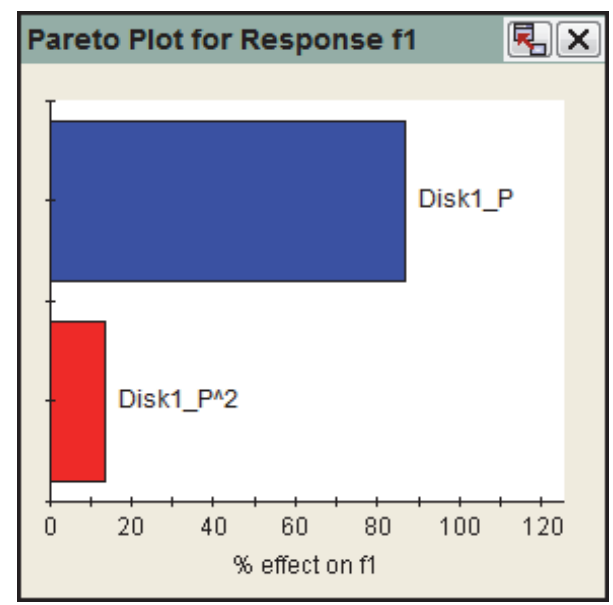

(c) Effect on $\mathrm{fl}$

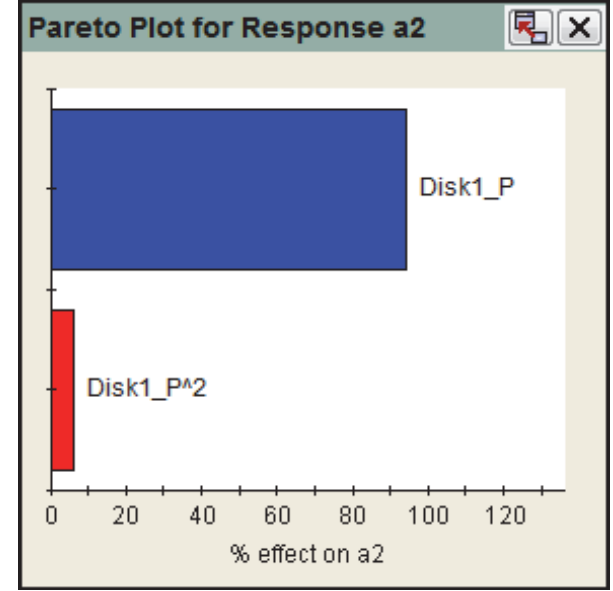

(b) Effect on a2

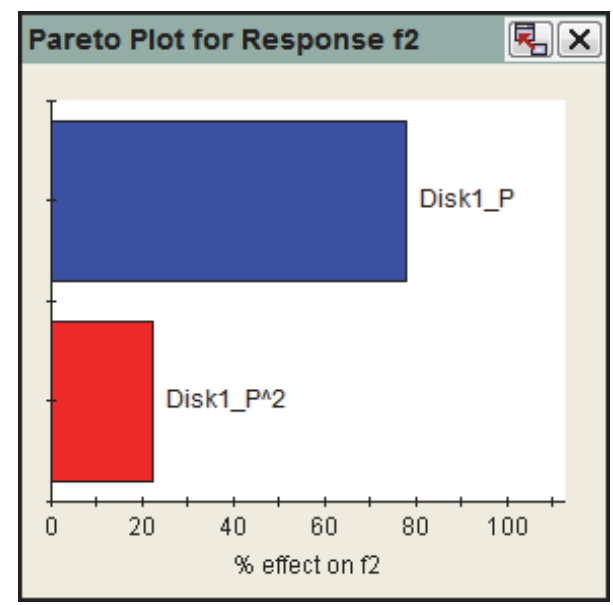

(d) Effect on $\mathrm{f} 2$

FIGURE 6: Effect of changing Disk1_P on optimization objectives.

TABLE 2: Experimental optimization result of Disk1_P and Disk2_P.

\begin{tabular}{lccccccc}
\hline & Disk1_P/m & Disk2_P/m & al/e $-4 \mathrm{~m}$ & $\mathrm{a} 2 / e-4 \mathrm{~m}$ & $\mathrm{v1} / \mathrm{V}$ & $\mathrm{v} 2 / \mathrm{V}$ & $\mathrm{cs} 1 /(\mathrm{r} / \mathrm{min})$ \\
\hline Baseline & 0.1835 & 0.332 & 1.79 & 1.485 & 3.43 & 4.35 & 2916 \\
After optimization & 0.178 & 0.324 & 1.114 & 1.317 & 2.45 & 3.7 & 2959 \\
\hline
\end{tabular}

after optimization. According to Figure 9, experimentally measured first-order critical speed of the rotor system before optimization is $2916 \mathrm{r} / \mathrm{min}$, while the numerical value according to Figure 3 is $2925.5 \mathrm{r} / \mathrm{min}$. The difference between the numerical simulation and experiment data is $0.33 \%$, validating the initial calculating model established in ANSYS.

Optimization result obtained from experimental data is shown in Table 2.

In Table 2, v1 represents the voltage of bearingl corresponding to $\mathrm{f} 1$, and $\mathrm{v} 2$ represents the voltage of bearing 2 corresponding to $\mathrm{f} 2$. The comparison between Tables 1 and 2 shows that the values of a1 and a 2 obtained from numerical simulation and experiment have little difference, which also validates the initial calculating model established in ANSYS.
According to Table 2, at the optimized positions of disk1 and disk 2 , the values of al and a 2 are decreased by $36 \%$ and $11 \%$, respectively; the values of v1 and v2 are decreased by $28.6 \%$ and $14.9 \%$, respectively. The optimization effectiveness of this strategy is significant.

As can be seen from the comparison between Tables 1 and 2, experimental reductions of the objectives are generally greater than numerical reductions. The main cause of this error is that the transient characteristics of the rotor-bearing system obtained by numerical calculation and experiments are not accurately consistent because the distribution of unbalance mass along the rotor is unknown [18]. Certain unbalance mass is imposed on the system in order to excite transient response when performing numerical calculation. 


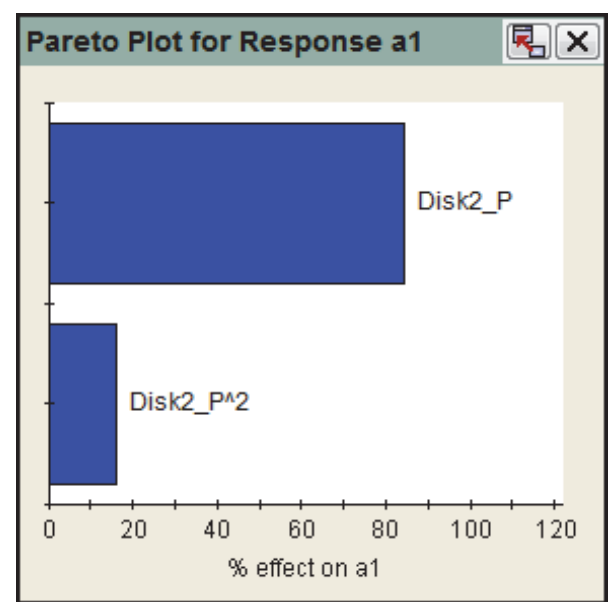

(a) Effect on al

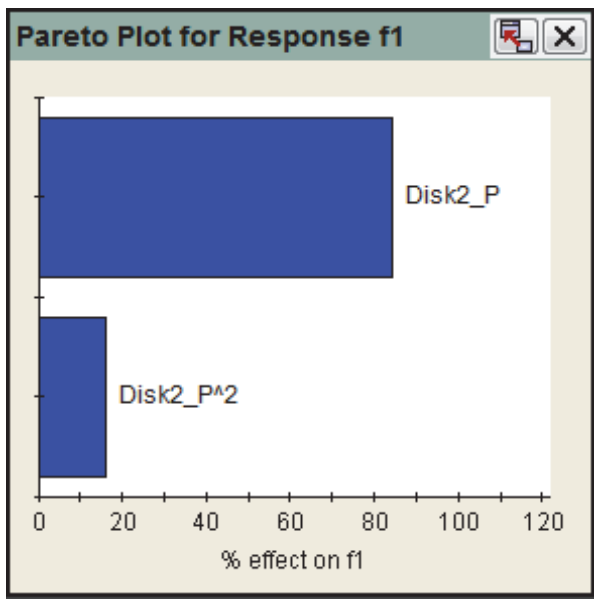

(c) Effect on $\mathrm{fl}$

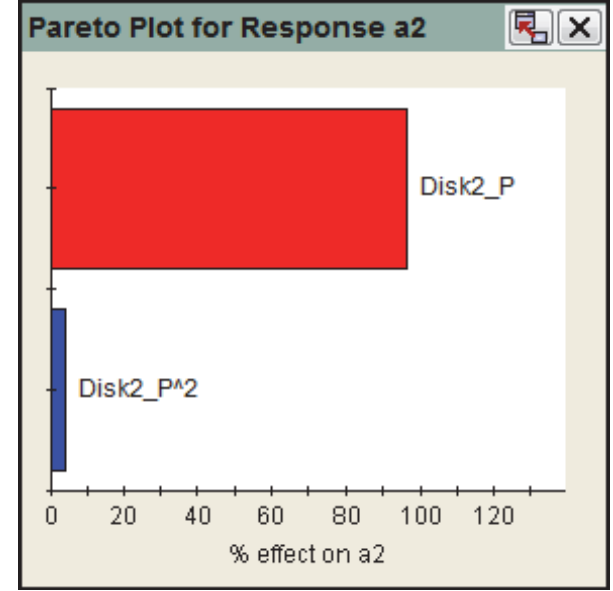

(b) Effect on a2

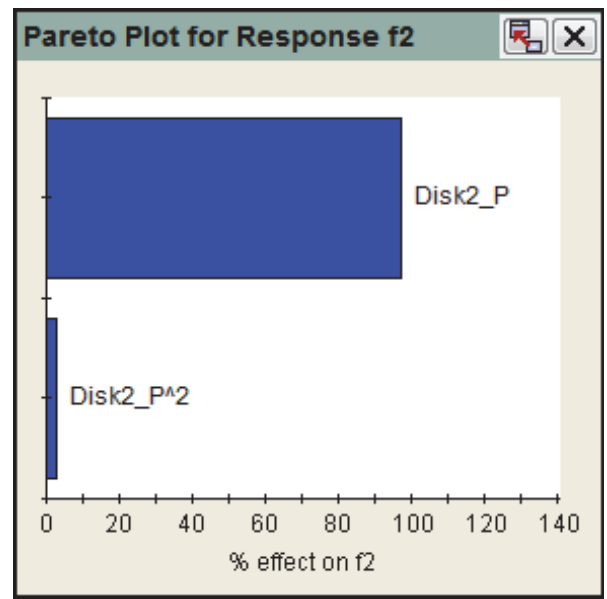

(d) Effect on f2

FIGURE 7: Effect of changing Disk2_P on optimization objectives.

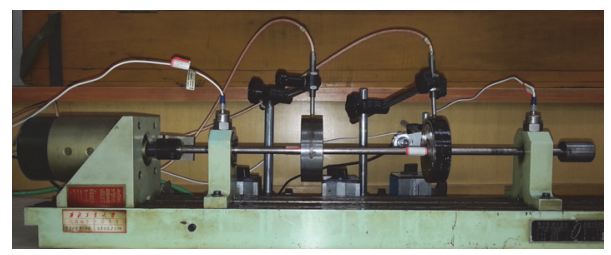

FIgURE 8: Test rig of the two-disk flexible rotor system.

In this paper, the unbalance mass on disk1 is assumed $25.5 \mathrm{~g} \cdot \mathrm{mm}$ at 195 degree, and the unbalance mass on disk 2 is assumed $17 \mathrm{~g} \cdot \mathrm{mm}$ at 57 degrees, while in the experiment the real distribution of unbalance mass is not like the assumption one. That is to say, the numerical results are obtained based on the hypothetical distribution of unbalance mass, while the experimental results are obtained based on the real distribution of unbalance mass. Much effort is needed to perform robust optimization based on the uncertainty of residual unbalance, which is our future work.

\section{Conclusions}

In this paper, a multiobjective optimization strategy is presented to reduce the vibration level of a flexible rotor system. A laboratory scale two-disk flexible rotor system is established and optimized using the proposed strategy. Experimental results of transient response before and after the optimization show that the vibration level of the rotor system is reduced significantly after optimization. The amplitudes of the two disks are reduced by $36 \%$ and $11 \%$, and 


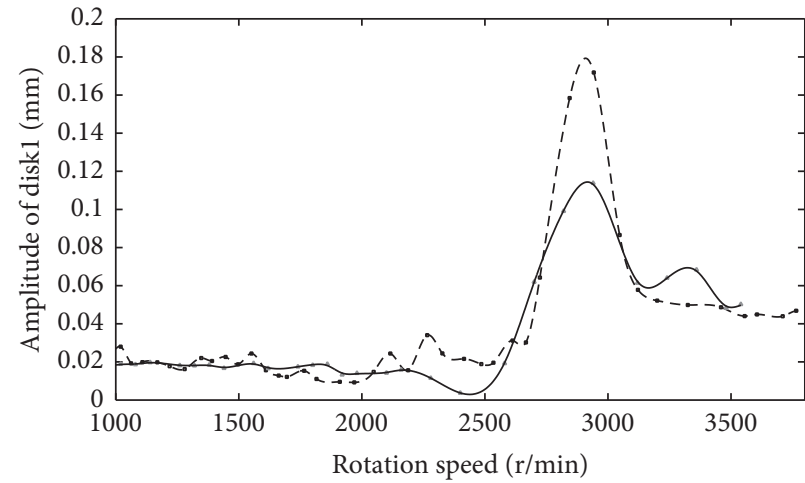

- Experimental data before optimization

- - - Fitting curve before optimization

- Experimental data after optimization

_ Fitting curve after optimization

(a) Transient response of al

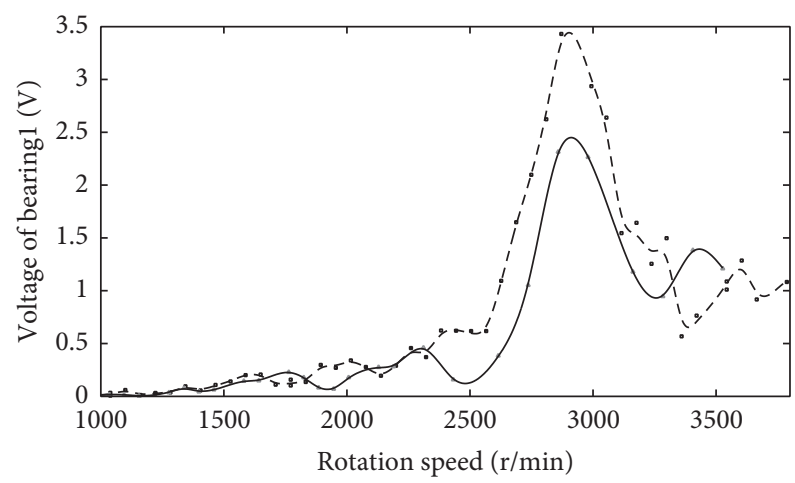

- Experimental data before optimization

- - - Fitting curve before optimization

- Experimental data after optimization

_ Fitting curve after optimization

(c) Transient response of f1

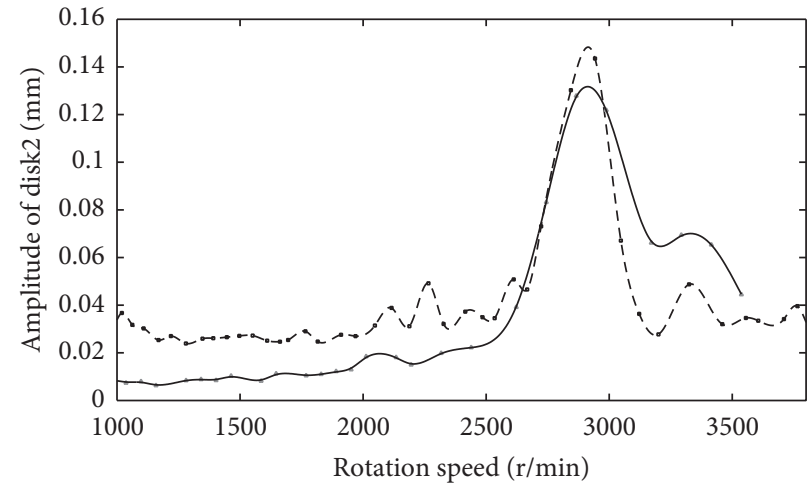

- Experimental data before optimization

- - - Fitting curve before optimization

- Experimental data after optimization

— Fitting curve after optimization

(b) Transient response of a2

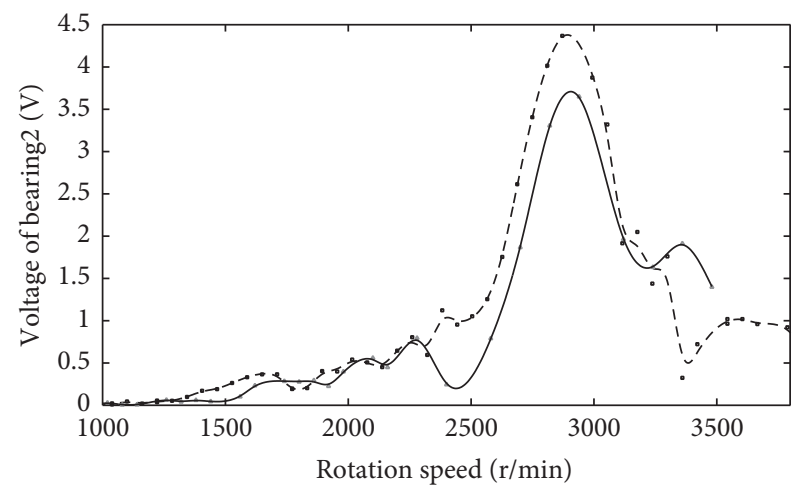

- Experimental data before optimization

- - - Fitting curve before optimization

- Experimental data after optimization

—_ Fitting curve after optimization

(d) Transient response of $\mathrm{f} 2$

FIGURE 9: Transient response before and after optimization of Disk1_P and Disk2_P.

the transmitted forces at the two bearings are reduced by $28.6 \%$ and $14.9 \%$, respectively. The experimental data supports the effectiveness of the proposed optimization strategy. The optimization strategy proposed in this paper can be an inspiration and reference for engineering rotor design.

\section{Competing Interests}

The authors declare that they have no competing interests.

\section{Acknowledgments}

This work was supported by the Aviation Industry Corporation of China (no. 51334050401).

\section{References}

[1] H. B. Zhang, Z. G. Li, and J. Chen, Design Manual of Aircraft Engine Machine, Aviation Industry Press, Beijing, China, 2000 (Chinese).

[2] T. P. Huang and G. L. Luo, "Dynamic optimization design of the rotor," Journal of Aerospace Power, vol. 9, no. 2, pp. 113-116, 1994 (Chinese).

[3] B. G. Choi and B. S. Yang, "Optimum shape design of rotor shafts using genetic algorithm," Journal of Vibration and Control, vol. 6, no. 2, pp. 207-222, 2000.

[4] D.-S. Lee and D.-H. Choi, "Reduced weight design of a flexible rotor with ball bearing stiffness characteristics varying with rotational speed and load," Journal of Vibration and Acoustics, vol. 122, no. 3, pp. 203-208, 2000.

[5] S.-C. Huang and C.-A. Lin, "Sensitivity analysis and optimization of undamped rotor critical speeds to supports stiffness," 
Journal of Vibration and Acoustics, Transactions of the ASME, vol. 124, no. 2, pp. 296-301, 2002.

[6] B. S. Yang, S. P. Choi, and Y. C. Kim, "Vibration reduction optimum design of a steam-turbine rotor-bearing system using a hybrid genetic algorithm," Structural and Multidisciplinary Optimization, vol. 30, no. 1, pp. 43-53, 2005.

[7] F. Strauß, M. Inagaki, and J. Starke, "Reduction of vibration level in rotordynamics by design optimization," Structural and Multidisciplinary Optimization, vol. 34, no. 2, pp. 139-149, 2007.

[8] D. H. Wang, Z. S. Liu, and W. Dou, "Design optimization of rotor system based on hybrid genetic algorithm," Journal of Vibration and Shock, vol. 28, no. 5, pp. 87-91, 2009 (Chinese).

[9] A. O. Pugachev, "Application of gradient-based optimization methods for a rotor system with static stress, natural frequency, and harmonic response constraints," Structural and Multidisciplinary Optimization, vol. 47, no. 6, pp. 951-962, 2013.

[10] T. N. Shiau, J. R. Chang, and W. B. Wu, "Multilevel optimization of the geared rotor-bearing system for multi-objectives with critical speed constraints," in Proceedings of the ASME Turbo Expo 2004 Power for Land, Sea, and Air, pp. 14-17, Vienna, Austria, June 2004.

[11] O. T. C. Matthew and S. K. Patrick, "On LMI-based optimization of vibration and stability in rotor system design," in Proceedings of the ASME Turbo. Expo. 2005: Power for Land, Sea and Air, pp. 6-9, Reno-Tahoe, Nev, USA, 2005.

[12] B.-K. Choi and B.-S. Yang, "Multiobjective optimum design of rotor-bearing systems with dynamic constraints using immunegenetic algorithm," Journal of Engineering for Gas Turbines and Power, vol. 123, no. 1, pp. 78-81, 2001.

[13] J. L. Gu, Rotor Dynamics, Defence Industry Press, Beijing, China, 1985.

[14] J.-J. Huang, L.-X. Zheng, G.-Q. Liu, and Q. Mei, “Optimization algorithm and experiment of a two-disk rotor system," Journal of Aerospace Power, vol. 31, no. 1, pp. 65-71, 2016 (Chinese).

[15] Y. Y. Lai, X. Jiang, and L. Q. Fang, Parameter Optimization Theory and Explain Examples with Isight, Beihang University Press, Beijing, China, 2012.

[16] D. H. Wang and Z. S. Liu, "Rotor structure optimal design based on genetic algorithm," Turbine Technology, vol. 47, no. 6, pp. 407-410, 2006 (Chinese).

[17] Y. E. Zhong, Rotor Dynamic, Tsinghua University Press, Beijing, China, 1987.

[18] C. D. Untaroiu and A. Untaroiu, "Constrained design optimization of rotor-tilting pad bearing systems," Journal of Engineering for Gas Turbines and Power, vol. 132, no. 12, Article ID 122502, 7 pages, 2010. 


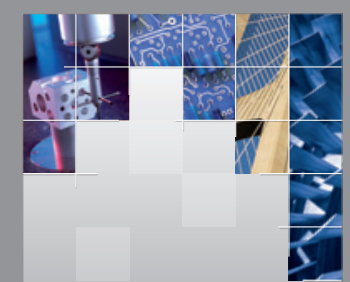

\section{Enfincering}
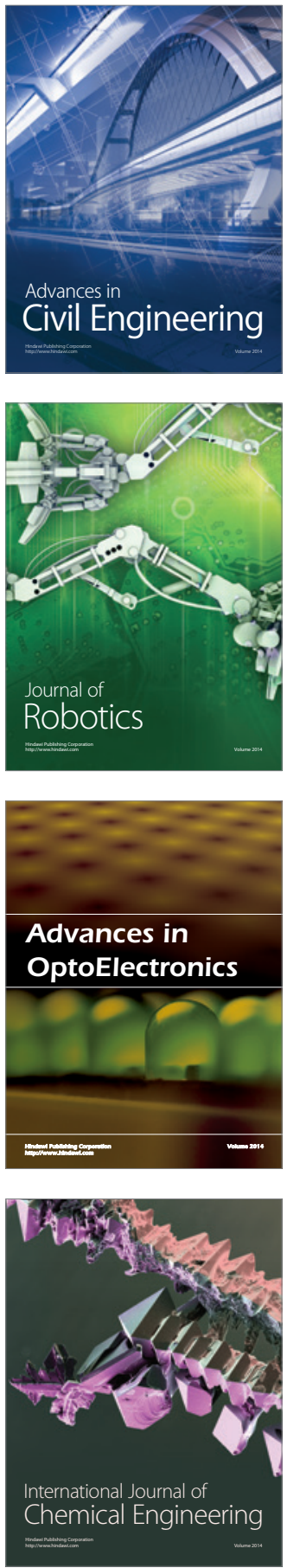

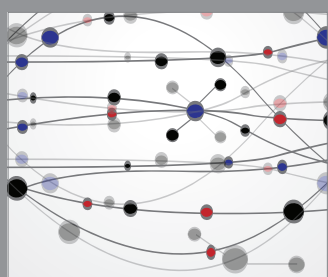

The Scientific World Journal

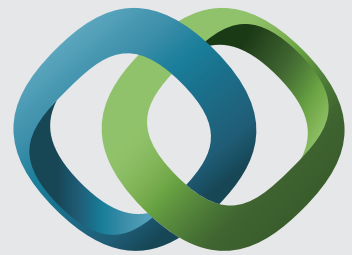

\section{Hindawi}

Submit your manuscripts at

https://www.hindawi.com
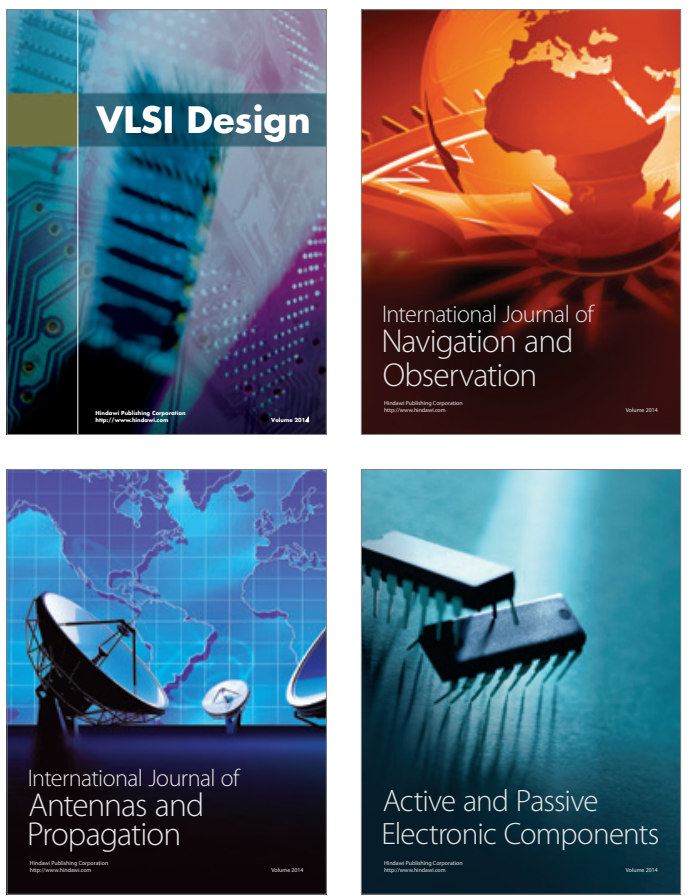
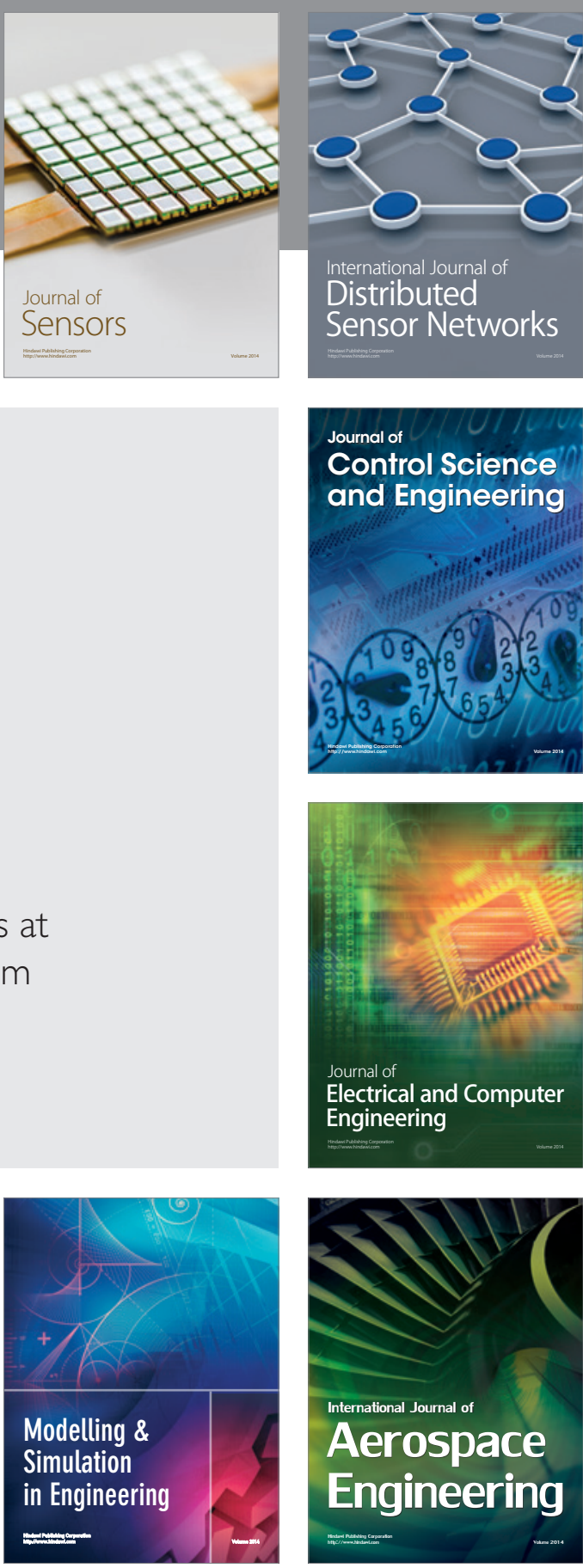

International Journal of

Distributed

Sensor Networks

$-$

Joumal of

Control Science

and Engineering
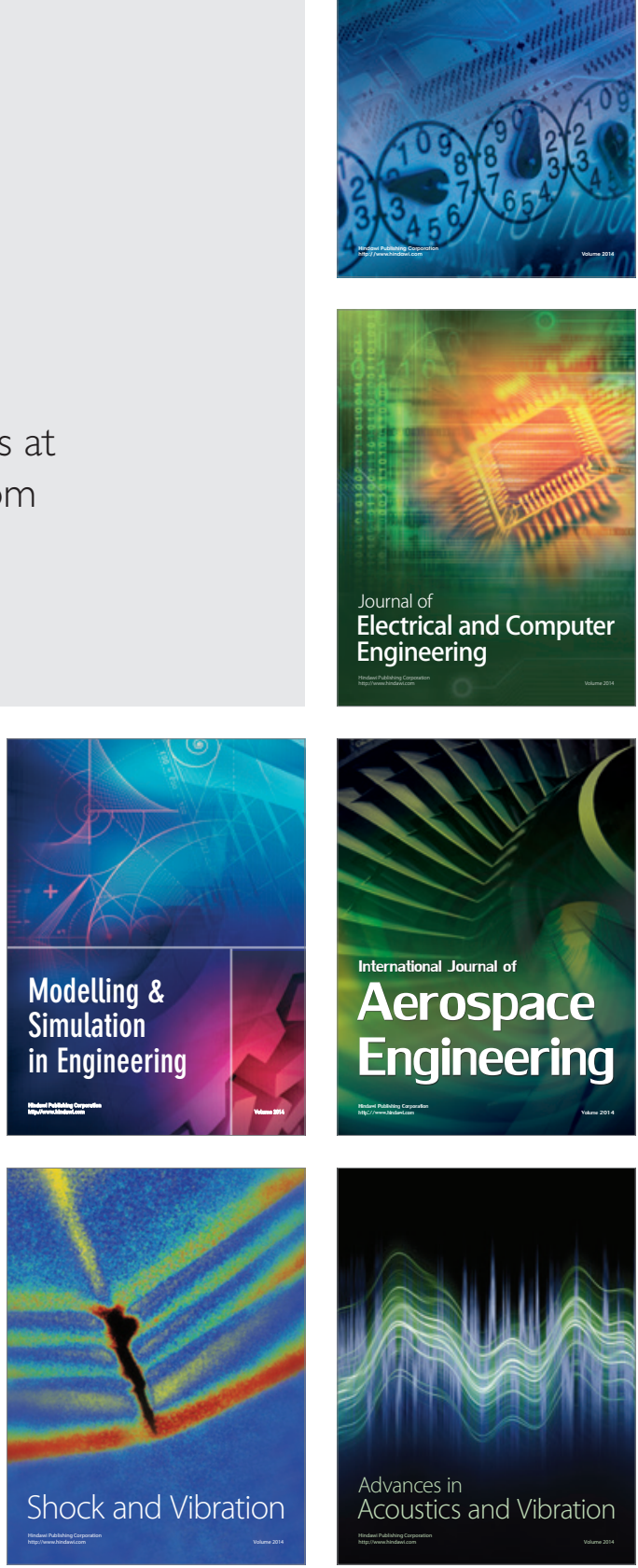